\title{
Rhinotrainer, Surgical Nose Simulator
}

Alberto Rancati ${ }^{\star}$, Daniel Moina, Gabriel Moina, Julio Dorr, Luis Ripetta and Santiago Horgon

University of Buenos Aires, Argentina

\begin{abstract}
The purpose of this paper is to present the Rhinotrainer, a surgical nose simulator. This innovative anatomical model is intended to facilitate the training of the nose surgeon. This opportunity provided by the Rhinotrainer enables the learning process and provides knowledge, which was previously available only through surgical practice and error.
\end{abstract}

Keywords: Rhinotrainer; Nose simulator; Rhinoplasty simulator; Rhinoplasty training; Neoderma; Surgical trainer; Open rhinoplasty; Closed rhinoplasty; Nasal tip sutures, Medical device; Anatomical model; Surgical model; Nasal approaches; Osteotomies; Nasal grafts.

\section{Introduction}

Rhinoplasty is a procedure that demands considerable experience of the surgeons who practice it, because the outcomes include not only the desired aesthetic appearance but also functional support and projection of the nasal appendix. To achieve these skills, constant practice is required of those who train as nose surgeons. A lack of cadaveric material on which to practice, should only be replaced by a nose simulator for patient safety [1].

The Rhinotrainer created by (Prodelphus Co. Brasil) (Figure 1) is a new generation, realistic simulator that recreates nasal tissues as if they were real. Thus, future rhinoplasty surgeons can access an anatomical substrate to develop various maneuvers that accelerate learning [2]. A number of principles also exist that, when respected and adopted, can lead to advanced learning in addition to predictable and favorable results. However, to accomplish this, the combination of technical skills, personal knowledge of the surgical technique, and experience through continuous practice is important.

The Rhinotrainer was developed with a material that simulates human tissues called Surgical Neoderma, which was previously used successfully in the Mastotrainer [3].

The Neoderma allows more than 60 types of consistencies, from mucosa to cartilage. Its materials accept various colorations to help the practitioner identify and anatomically differentiate various tissues. This tool belongs to a new generation of simulators called Realistic Endo Surgical Trainers (R.E.S.T.), which represent a change in surgical education [4].

Surgeons can practice as often as needed, improving their experience

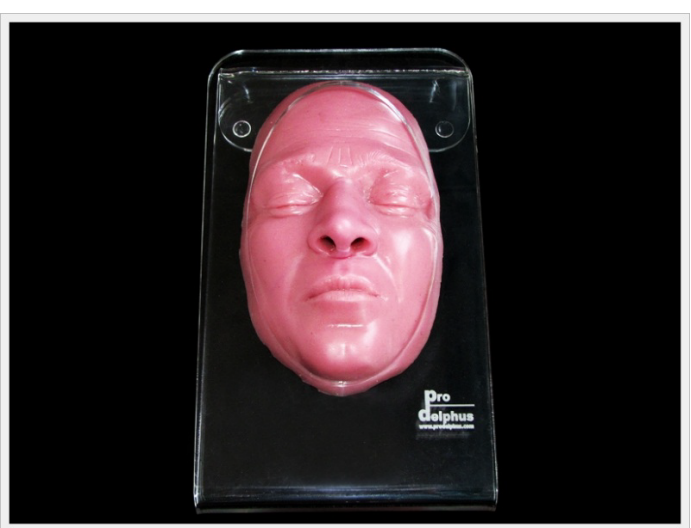

Figure 1: Rhinotrainer. and skills in less time. Furthermore, there is reduced risk and stress [5].

While surgical errors are and will be unavoidable, it is preferable to first experience surgery on a simulator rather than a patient. The systematic practice of strategies and maneuvers in a simulation program can significantly contribute to risk management and patient safety.

In this paper, we discuss the different applications that can be performed on the simulator.

\section{A practical Guide to Preoperative Analysis}

\section{Inspection and Palpation}

A thorough preoperative analysis, leading to a correct diagnosis of preexisting nasal deformity, is of fundamental importance to assess the aesthetic and functional alterations of the nose in each patient.

Through inspection, palpation (Figure 2) and nasal endoscopy, one can determine which structural elements are responsible for the alterations affecting the patient. In this way, the surgical plan can be created by external and internal palpation [6].

\section{Different markings}

Knowledge of the surgical anatomy is necessary for any surgeon.

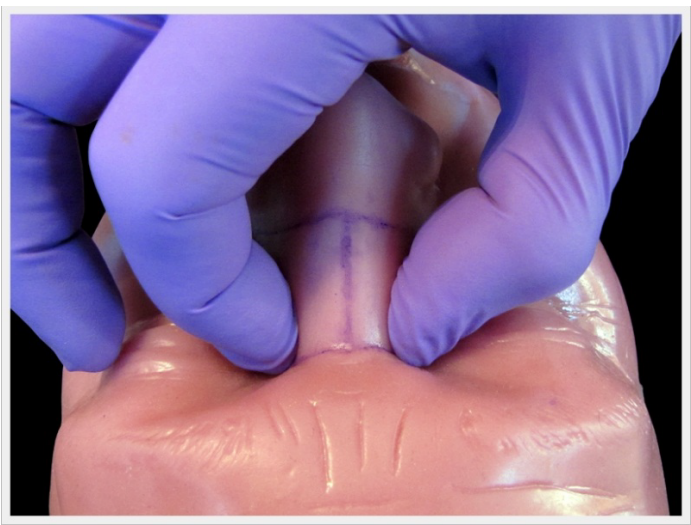

Figure 2: Inspection and palpation of the nose.

*Corresponding author: Alberto Rancati, University of Buenos Aires, Buenos Aires, Argentina, Tel: +54-147877500; E-mail: Rancati@gmail.com

Received July 01, 2014; Accepted September 23, 2014; Published September 30, 2014

Citation: Rancati A, Moina D, Moina G, Dorr J, Ripetta L (2014) Rhinotrainer Surgical Nose Simulator. Surgery Curr Res 4: 205. doi:10.4172/2161-1076.1000205

Copyright: @ 2014 Rancati $A$, et al. This is an open-access article distributed under the terms of the Creative Commons Attribution License, which permits unrestricted use, distribution, and reproduction in any medium, provided the original author and source are credited. 
Citation: Rancati A, Moina D, Moina G, Dorr J, Ripetta L (2014) Rhinotrainer, Surgical Nose Simulator. Surgery Curr Res 4: 205. doi:10.4172/21611076.1000205

The simulators enable surgeons to exercise the different incisions of the rhinoplasty approach (open or closed) and anatomical repairs. They may be marked for better understanding, with the possibility to delete and re-mark as many times as necessary (Figure 3).

\section{Infiltration}

Various infiltration techniques (Figure $4 \mathrm{a}$ and $4 \mathrm{~b}$ ) can be taught, considering the chosen approach, anatomical planes, and elements. [7].

\section{Methods of approach}

Different techniques and surgical approaches (Figure $5 \mathrm{a}$ and $5 \mathrm{~b}$ ) can be practiced [8], remembering that the anatomy, planning, and surgical technique are unique for each patient. "No surgical technique will prepare the surgeon to the different cases".

Because of the composition of the material in the simulator, the material can be incised with cold, electrical, and laser scalpels.

Detachment, exposure, and identification of the triangular and quadrangular alar cartilages and nasal bones: The dissection is performed with blunt scissors (Figure 6a) for exposure of the nasal

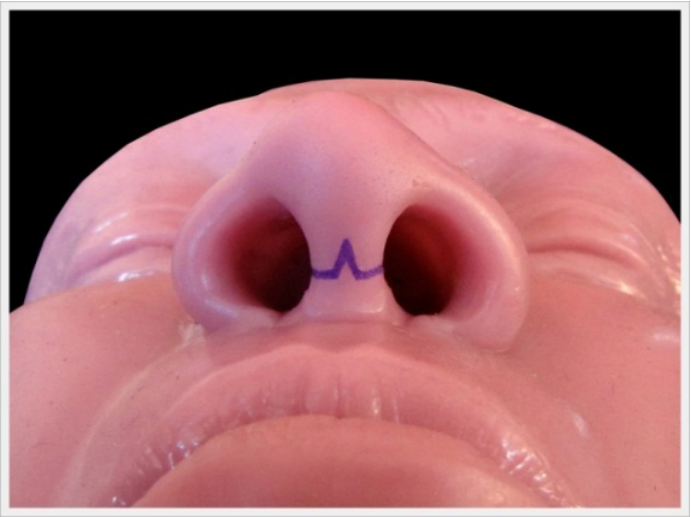

Figure 3: Different markings on the skin

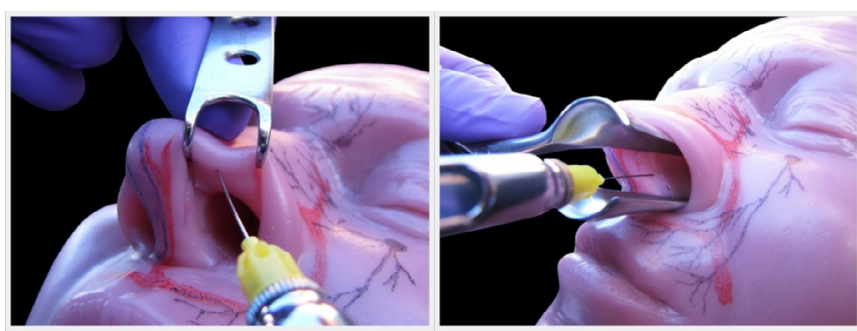

Figure 4a, b: Local anesthesia.

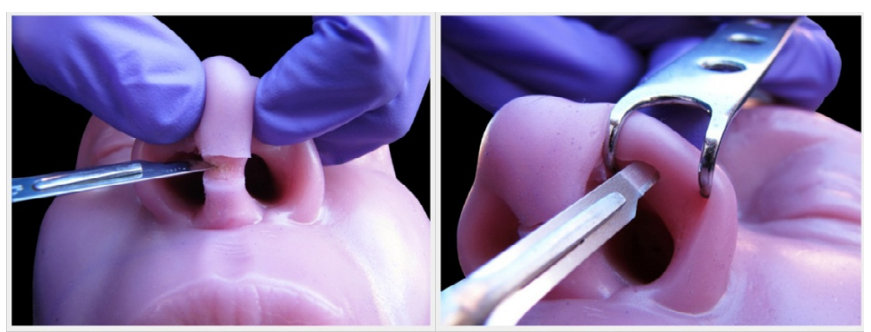

Figure 5a, b: Incisions and approaches.

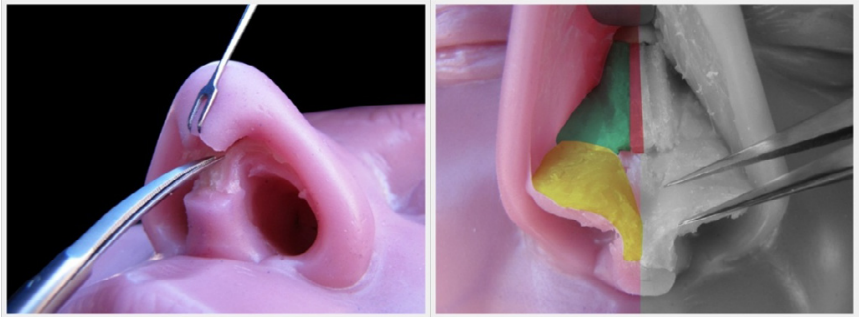

Figure 6a,b: Exposure and identification of nasal structures.
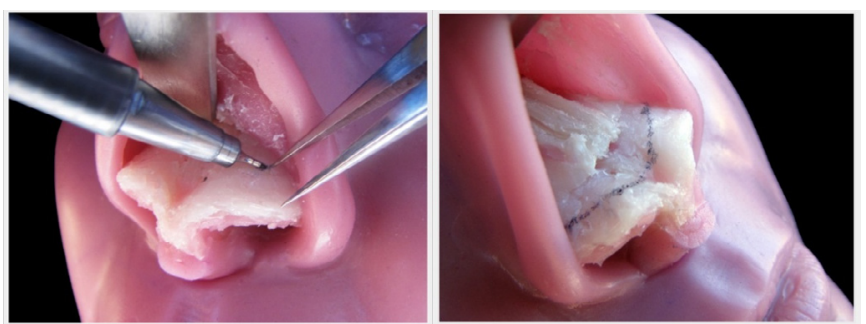

Figure 7a, b: Marking the alar cartilages
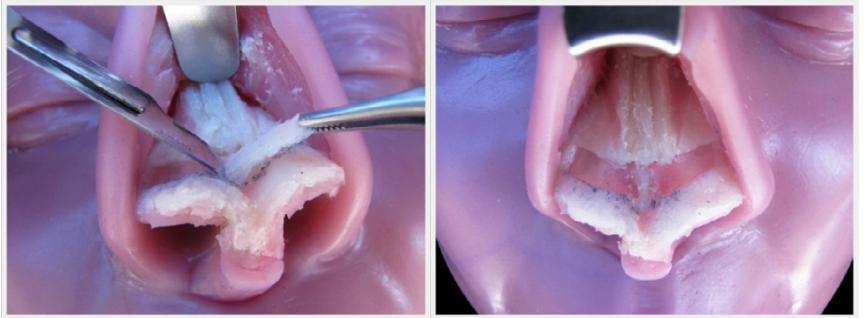

Figure 8a, b: Resection of the cephalic part of the lateral crus.

structures (observed as digitally colored for better interpretation and comprehension in Figure 6b).

Marking, carving (Figure $7 \mathrm{a}$ and $7 \mathrm{~b}$ ), and resection (Figure 8a and $8 b$ ) of the alar or lateral inferior cartilages depending on the surgical technique: Nasal aesthetic surgery is called "the millimeter surgery" because small mistakes will have a major impact on the final result. With the simulator, the surgeon can refine his surgical technique and gain more skills and abilities to obtain better and longer lasting results.

\section{Resection of the bony and cartilaginous hump}

The cartilaginous dorsum can be treated using a scalpel or scissors (Figure 9a) depending on the technique. $9 b)$.

The bony hump may be reduced with a rasp or osteotome (Figure

The nasal bones and resected cartilages rest on the nasal dorsum (digitally colored for better distinction in Figure 10a). The alignment of the bony dorsum is achieved with a rasp (Figure 10b).

Internal or external osteotomies: The bony pyramid is mobilized and repositioned with internal or external osteotomies (Figure 11).

Preparation and placement of grafts: Within this simulator, one can carve various types of cartilaginous grafts using the Synthetic Nose 
Citation: Rancati A, Moina D, Moina G, Dorr J, Ripetta L (2014) Rhinotrainer, Surgical Nose Simulator. Surgery Curr Res 4: 205. doi:10.4172/21611076.1000205

Cartilage Simulator for use in primary and secondary rhinoplasties (Figure 12a and 12b).

The material, which is similar to cartilage, allows training with different grafts such as columellar struts (Figure 13a) [9], shields (Figure 13b) [10], spreader grafts [11], alar batten [12], and dorsum grafts.

Suture: Owing to the various resistances of the simulator (e.g., skin, cartilage), the surgeon can practice not only the approach of the closure incisions but also the placement of different grafts (Figure 14a

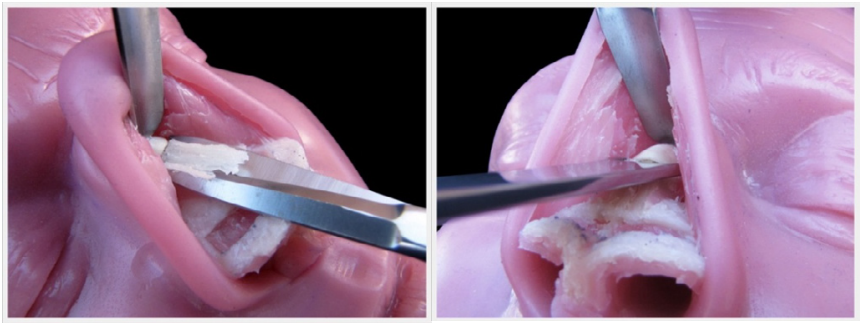

Figure 9a, b: Nasal hump removal.

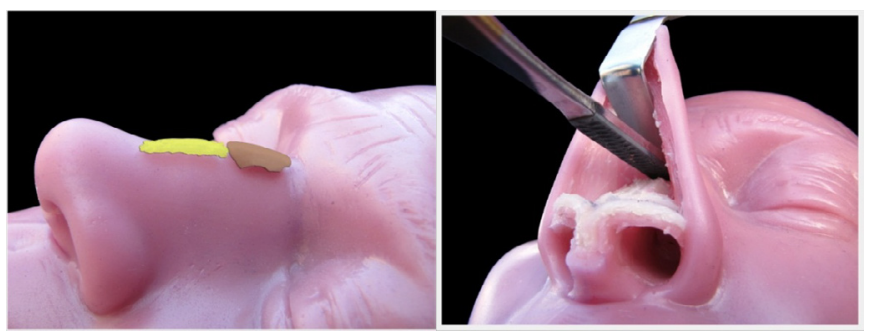

Figure $10 a$, b: The bony dorsum is aligned using a rasp.

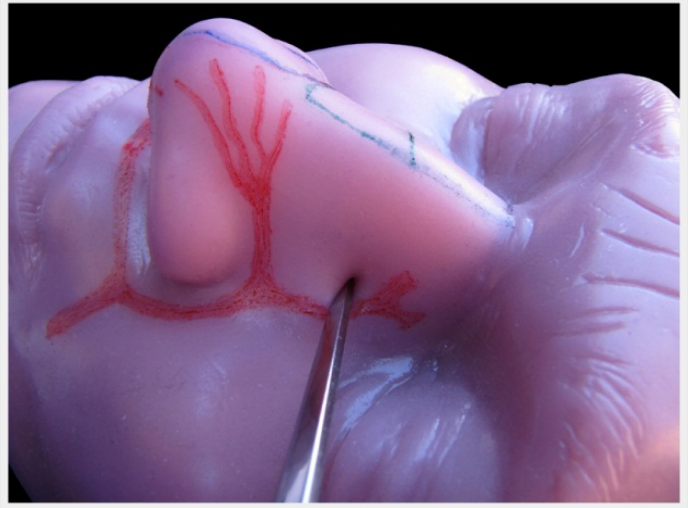

Figure 11: External osteotomy.
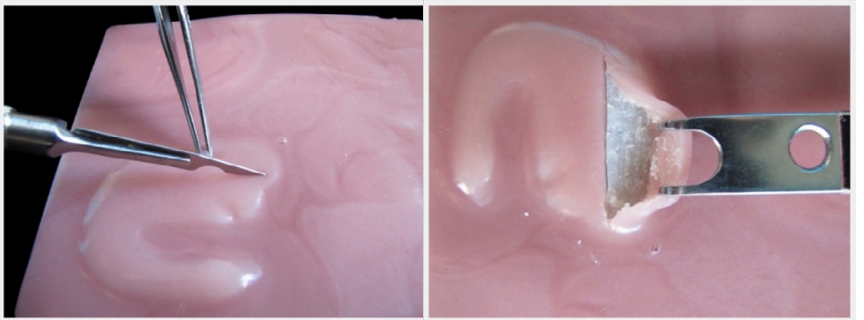

Figure 12a, b: Various types of cartilaginous grafts are available for carving.
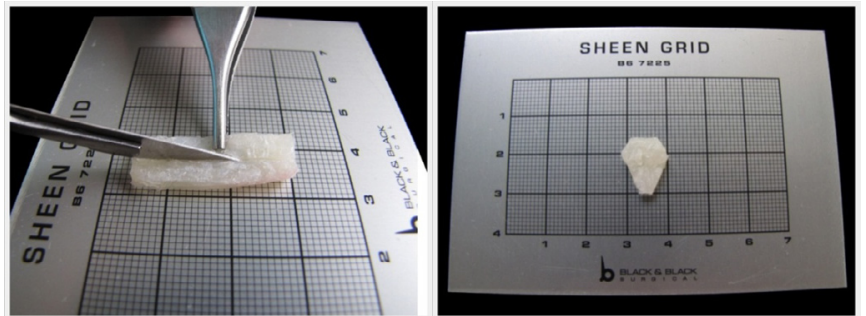

Figure 13a, b: Columellar struts and shields are seen.
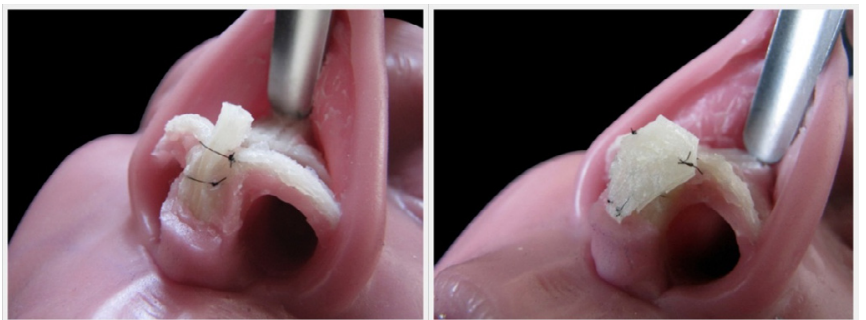

Figure 14a, b: Different grafts can be placed.

and $14 \mathrm{~b})$. The surgeon can access carved cartilages previously extracted from the simulator and the stitches needed to shape the cartilage that will determine the new shape of the nose.

\section{Conclusion}

The use of simulators promotes a shift in the theoretical-practical preparation of surgeons and the development and training of new surgical techniques. The purpose of this simulator is to reduce the learning curve for surgeons who are new to the complex world of rhinoplasty, to gain surgical experience in a harmless environment, and to reduce personal and institutional liability.

The Rhinotrainer hás been specially designed for training in the aesthetic and reconstructive field; and validation techniques followed by precision assessments are in course to attest the model's feasibility.

\section{Conflict of Interest}

All authors have no conflicts of interest to disclose in relation to this article.

\section{References}

1. Dutta S, Krummel TM (2006) Simulation: a new frontier in surgical education. Adv Surg 40: 249-263.

2. Zymberg S, Vaz-Guimarães Filho F, Lyra M (2010) Neuroendoscopic training presentation of a new real simulator. Minim Invasive Neurosurg 53: 44-46.

3. Matthes AG, Perin LF, Rancati A, da Fonseca L, Lyra M (2012) Mastotrainer new training project for breast aesthetic and reconstructive surgery. Plas Reconstr Surg 130: 502e-4e.

4. Choy I, Okrainec A (2010) Simulation in surgery: perfecting the practice. Surg Clin North Am 90: 457-473.

5. Franzese $\mathrm{CB}$, Stringer SP (2007) The evolution of surgical training: perspectives on educational models from the past to the future. Otolaryngol Clin North Am 40: 1227-1235, vii.

6. Hinderer KH (1971) Fundamentals of anatomy and surgery of the nose Birmingham, Ala: Aesculapius Pub Co, USA.

7. Tom L, Tardy ME (1984) Anaesthesia in rhinoplasty. Facial Plast Surg 1: 2.

8. Aiach G, Madjiji A, Horn G, Sheen JH (1996) Atlas of Rhinoplasty: Open and Endonasal Approaches. (1 ${ }^{\text {st }}$ edn) Quality Med Pub, St Louis.

9. Rohrich RJ, Hoxworth RE, Kurkjian TJ (2012) The role of the columellar strut in rhinoplasty: indications and rationale. Plast Reconstr Surg 129: 118e-125e. 
Citation: Rancati A, Moina D, Moina G, Dorr J, Ripetta L (2014) Rhinotrainer, Surgical Nose Simulator. Surgery Curr Res 4: 205. doi:10.4172/21611076.1000205

10. Kamer FM, Churukian MM (1984) Shield graft for the nasal tip. Arch Otolaryngol 110: 608-610.

11. Sheen JH (1984) Spreader graft: a method of reconstructing the roof of the middle nasal vault following rhinoplasty. Plast Reconstr Surg 73: 230-239.

12. Becker DG, Becker SS (2003) Treatment of nasal obstruction from nasal valve collapse with alar batten grafts. J Long Term Eff Med Implants 13: 259-269. 